\title{
Excluded-Volume Effects in Star Polymer Solutions. Six-Arm Star Polystyrene in Benzene
}

\author{
Mitsuhiro Okumoto, Yoshimi Iwamoto, Yo NaKamura, and Takashi NorisuYe
}

Department of Macromolecular Science, Osaka University,

1-1 Machikaneyama-cho, Toyonaka, Osaka 560-0043, Japan

(Received November 17, 1999)

\begin{abstract}
Seven narrow-distribution samples of six-arm star polystyrene ranging in weight-average molecular weight $M_{w}$ from $6.1 \times 10^{4}$ to $3.4 \times 10^{6}$ in benzene at $25^{\circ} \mathrm{C}$ have been studied by light scattering and viscometry to determine their $z$-average radii of gyration, second and third virial coefficients, and intrinsic viscosities. The ratios of the respective properties to those of linear polystyrene in the same solvent are established for high $M_{w}$. Data analysis shows that the relation between the radius expansion factor and the conventional excluded-volume parameter $z$ comes close to the known relations for four-arm star and linear polystyrenes of high molecular weight and is described fairly satisfactorily by the previously proposed interpolation formula. On the other hand, the viscosity expansion factor vs. $z$ curve appears slightly below that for linear polystyrene though almost superimposed on that for the four-arm star polymer. Thus the difference in this expansion factor between the linear and star chains remains to be explained theoretically. The experimental interpenetration function for the six-arm star polymer gradually decreases to about 0.6 , a value close to recent Monte Carlo data, with increasing $M_{w}$. Its comparison with the previously constructed interpolation expression suggests that, as was the case for linear flexible polymers, the effect of chain stiffness on the second virial coefficient needs to be considered for $M_{w}$ below $10^{6}$.

KEY WORDS Star Polymer / Polystyrene / Excluded-Volume Effect / Light Scattering / Expansion Factor/Interpenetration Function /
\end{abstract}

Much work has been done on star polymer solutions to clarify the effects of molecular architecture on dilute solution properties, ${ }^{1-3}$ but even for stars consisting of relatively small arm numbers excluded-volume effects still remain to be investigated. The present work is concerned with those effects on the mean-square radius of gyration $\left\langle S^{2}\right\rangle$, the second virial coefficient $A_{2}$, the third virial coefficient $A_{3}$, and the intrinsic viscosity [ $\eta$ ] for six-arm star polystyrene in benzene, a good solvent. It is an extension of our previous light scattering and viscosity studies on this polymer in cyclohexane ${ }^{4}$ at and near $\Theta$ and on four-arm star polystyrene in cyclohexane and benzene. ${ }^{5,6}$ Its major purpose is two-fold. One is to determine the ratio of each of $\left\langle S^{2}\right\rangle_{z}$ (the $z$-average $\left\langle S^{2}\right\rangle$ ), $A_{2}, A_{3}$, and $[\eta]$ for the six-arm star polymer in the good solvent to that for the linear chain with the same molecular weight. The other is to test our previously proposed interpolation formulas ${ }^{5}$ for the radius expansion factor $\alpha_{S}\left[\equiv\left(<S^{2}>/<S^{2}>_{0}\right)^{1 / 2}\right]$ and the interpenetration function $\Psi$ appearing in $A_{2}$, where $\left\langle S^{2}\right\rangle_{0}$ denotes the unperturbed $\left\langle S^{2}\right\rangle$. With regard to the second purpose, the following remarks may be pertinent here.

The interpolation formulas ${ }^{5}$ for $\alpha_{S}^{2}$ and $\Psi$ were constructed in the binary cluster approximation by combining the respective first-order perturbation theories ${ }^{7,8}$ with the Monte Carlo data in the self-avoiding limit. 9,10 The use of this approximation is based on our pervious finding ${ }^{5}$ that the effects of ternary cluster interactions on $\left\langle S^{2}>\right.$ and $A_{2}$ for four-arm star polystyrene in cyclohexane at the $\Theta$ temperature are negligible in the range of weight-average molecular weight $M_{w}$ (from $8.5 \times 10^{4}$ to $3.1 \times 10^{6}$ ) studied if the binary cluster integral is replaced by a linear combination of the binary and ternary cluster integrals. ${ }^{11}$ For six-arm star polystyrene in the $\Theta$ solvent, the residual ternary effects on $\left\langle S^{2}>\right.$ and $A_{2}$ ap- peared to remain as predicted by perturbation calculations, ${ }^{5,12}$ but they were not so significant as to impair the binary cluster approximations to $\alpha_{S}{ }^{2}$ and $\Psi^{4}{ }^{4}$

\section{EXPERIMENTAL}

\section{Polymer Samples}

The previously investigated samples ${ }^{4}$ of six-arm star polystyrene, $6 \mathrm{~S} 10,6 \mathrm{~S} 17,6 \mathrm{~S} 36,6 \mathrm{~S} 85,6 \mathrm{~S} 190,6 \mathrm{~S} 260$, and $6 \mathrm{~S} 570$, were used for the present study. They had been prepared by anionic polymerization followed by fractional precipitation. The ratio of $M_{w}$ for each sample to that for its arm was $6 \pm 0.3$. The weight-average to number-average molecular weight ratios estimated by gel permeation chromatography were less than 1.04 for samples $6 \mathrm{~S} 17,6 \mathrm{~S} 36,6 \mathrm{~S} 85,6 \mathrm{~S} 190$, and $6 \mathrm{~S} 260{ }^{4}$

\section{Light Scattering}

Intensities of light scattered from six-arm polystyrene in benzene at $25^{\circ} \mathrm{C}$ were measured on a Fica-50 light scattering photometer with vertically polarized incident light of 436-nm wavelength in an angular range from 30 to $150^{\circ} \mathrm{C}$. As in our previous work, ${ }^{4}$ benzene at $25^{\circ} \mathrm{C}$ was used to calibrate the apparatus. Seven solutions of different polymer concentrations were studied for each sample. The polymer mass concentration $c$ was calculated from the weight fraction of the solute with the solution density approximated by the solvent density. This approximation did not introduce errors greater than $0.3 \%$ in the values of $A_{2}$ and $A_{3}$.

The specific refractive index increment $\partial n / \partial c$ of sixarm star polystyrene in benzene at $25^{\circ} \mathrm{C}$ and $436 \mathrm{~nm}$ was measured for all of the seven samples using a modified Schulz-Cantow differential refractometer. It was a weakly increasing function of $M_{w}$ represented by 


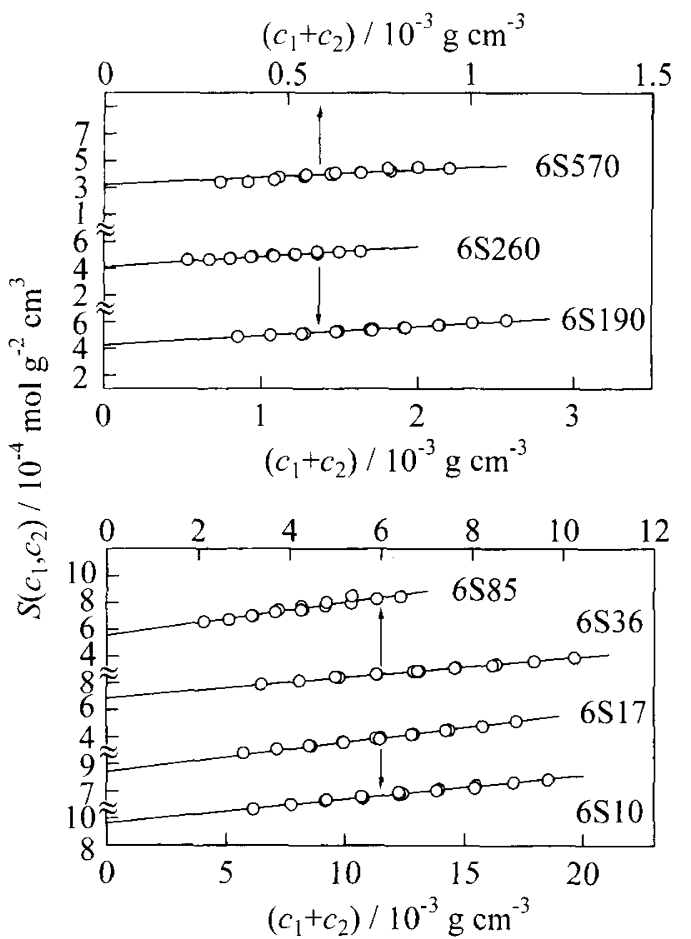

Figure 1. Plots of $S\left(c_{1}, c_{2}\right)$ vs. $c_{1}+c_{2}$ for the indicated samples of six-arm star polystyrene in benzene at $25^{\circ} \mathrm{C}$.

$$
\partial n / \partial c=-217 / M_{w}+0.1075\left(\mathrm{~cm}^{3} \mathrm{~g}^{-1} ; 436 \mathrm{~nm}\right)
$$

\section{Viscometry}

Intrinsic viscosities for all samples in benzene at $25^{\circ} \mathrm{C}$ were determined by conventional capillary viscometers of the Ubbelohde type.

\section{RESULTS}

\section{Analysis of Light-Scattering Data}

Excess reduced scattering intensities $R_{\theta}$ at scattering angles $\theta$ were extrapolated to $\theta=0$ to obtain $R_{0}$ (i.e., $R_{\theta}$ at $\theta=0)$, with the aid of the square-root plot ${ }^{13}$ of $(K c /$ $\left.R_{\theta}\right)^{1 / 2} v s \cdot \sin ^{2}(\theta / 2)$ for all samples except the highest molecular weight one, $6 \mathrm{~S} 570$ ( $K$ denotes the optical constant). This type of plot for $6 \mathrm{~S} 570$ bent upward for $\sin ^{2}$ $(\theta / 2)$ larger than 0.4 at any $c$ studied and the extrapolation for the sample was facilitated by plotting $\left(K c / R_{\theta}\right)^{1 / 4}$ against $\sin ^{2}(\theta / 2)$. According to our previous analysis ${ }^{6}$ of the particle scattering function for Gaussian star chains composed of $f$ identical arms, the plot of $\left(K c / R_{0}\right)^{1 / p} v s$. $\sin ^{2}(\theta / 2)$ follows the initial tangent over the widest $\theta$ if $p$ is taken as $64 / 17$, a value close to 4 , for $f=6$.

The data for $K c / R_{0}$ obtained were analyzed according to the equation ${ }^{14,15}$

$$
\begin{aligned}
S\left(c_{1}, c_{2}\right) & \equiv \frac{\left(K c / R_{0}\right)_{c=c_{2}}-\left(K c / R_{0}\right)_{c=c_{1}}}{c_{2}-c_{1}} \\
& =2 A_{2}+3 A_{3}\left(c_{1}+c_{2}\right)+\cdots
\end{aligned}
$$

where $\left(K c / R_{0}\right)_{c=c_{1}}$ and $\left(K c / R_{0}\right)_{c=c_{2}}$ denote the values of $K c /$ $R_{0}$ at different concentrations $c_{1}$ and $c_{2}$, respectively. The plots of $S\left(c_{1}, c_{2}\right)$ vs. $c_{1}+c_{2}$ constructed are shown in Fig-

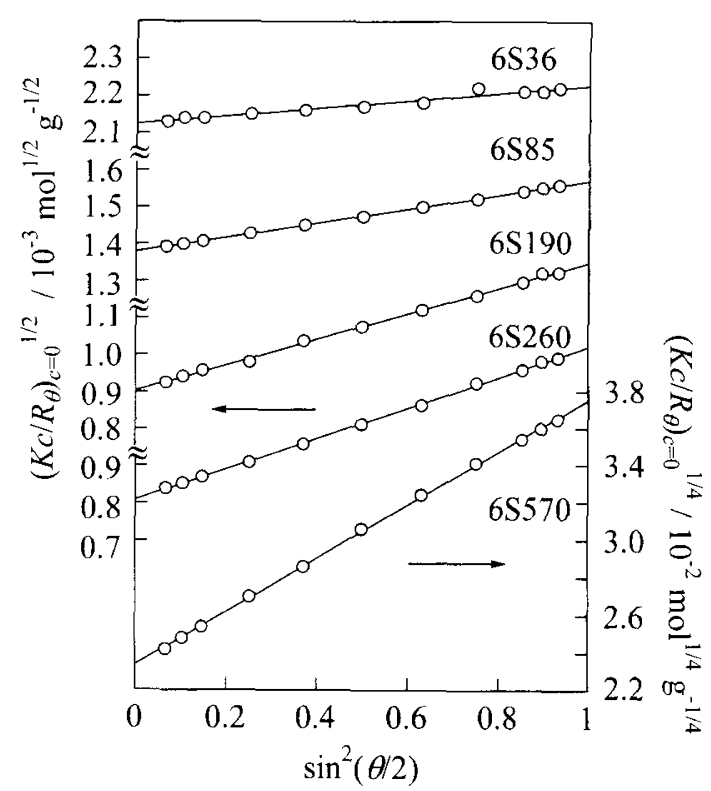

Figure 2. Angular dependence of $\left(K c / R_{\theta}\right)_{c=0}{ }^{1 / 2}$ for samples $6 \mathrm{~S} 36$, $6 \mathrm{~S} 85,6 \mathrm{~S} 190$, and $6 \mathrm{~S} 260$ and that of $\left(K_{c} / R_{\theta}\right)_{c-0}{ }^{1 / 4}$ for sample $6 \mathrm{~S} 570$, all in benzene at $25^{\circ} \mathrm{C}$.

Table I. Results from light scattering and viscosity measurements on six-arm star polystyrene samples in benzene at $25^{\circ} \mathrm{C}$

\begin{tabular}{lcccccc}
\hline Sample & $10^{-4} M_{w}$ & $\frac{10^{4} A_{2}}{\mathrm{~cm}^{3} \mathrm{~mol} \mathrm{~g}^{-2}}$ & $\frac{10^{2} A_{3}}{\mathrm{~cm}^{6} \mathrm{~mol} \mathrm{~g}^{-3}}$ & & $\frac{\left\langle S^{2}\right\rangle_{z}^{1 / 2}}{\mathrm{~nm}}$ & $\frac{10^{-2}[\eta]}{\mathrm{cm}^{3} \mathrm{~g}^{-1}}$ \\
\hline $6 \mathrm{~S} 10$ & 6.07 & 4.82 & 0.58 & & 0.195 \\
$6 \mathrm{~S} 17$ & 10.1 & 4.21 & 0.63 & & 0.296 \\
$6 \mathrm{~S} 36$ & 21.9 & 3.43 & 0.93 & 12 & 0.475 \\
$6 \mathrm{~S} 85$ & 53.3 & 2.80 & 1.53 & 20.8 & 0.883 \\
$6 \mathrm{~S} 190$ & 119 & 2.13 & 2.3 & 33.5 & 1.66 \\
$6 \mathrm{~S} 260$ & 151 & 2.05 & 2.5 & 39.4 & 1.93 \\
$6 \mathrm{~S} 570$ & 335 & 1.60 & 4.3 & 61.3 & 3.80 \\
\hline
\end{tabular}

ure 1 , where the data for pairs of neighboring $c_{1}$ and $c_{2}$ in a series of polymer concentrations have been omitted, since they were less accurate. The plotted points for each sample follow a straight line whose intercept and slope give $A_{2}$ and $A_{3}$, respectively.

With the virial coefficients thus obtained, the apparent molecular weight $M_{\text {app }}$ defined by $M_{\text {app }} \equiv\left(K c / R_{0}-\right.$ $\left.2 A_{2} c-3 A_{3} c^{2}\right)^{-1}$ was calculated for each sample, and $M_{w}$ was evaluated by its extrapolation to infinite dilution. The plot of $M_{\text {app }} v s$. $c$ was essentially horizontal for any sample, facilitating the extrapolation.

Values of $\left(K c / R_{\theta}\right)_{c=0}$, i.e., infinite-dilution values of $K c / R_{\theta}$, were obtained by extrapolation of $\left(K c / R_{\theta}\right)^{1 / 2}$ plotted against $c$ at fixed $\theta$. Figure 2 shows the angular dependence of $\left(K c / R_{\theta}\right)_{c=0}{ }^{1 / 2}$ or $\left(K c / R_{\theta}\right)_{c=0}{ }^{1 / 4}$ (for sample $6 \mathrm{~S}$ 570 ), from which $\left\langle S^{2}\right\rangle_{z}$ for each sample was determined. The numerical results of $M_{w}, A_{2}, A_{3}$, and $\left\langle S^{2}\right\rangle_{z}$ are summarized in Table I, along with those of $[\eta]$.

Molecular Weight Dependence of $\left\langle S^{2}\right\rangle_{z}, A_{2}, A_{3}$, and [ $\left.\eta\right]$

Our $\left\langle S^{2}\right\rangle_{z}$ data for six-arm star polystyrene (unfilled circles) in benzene at $25^{\circ} \mathrm{C}$ are compared with those for four-arm $\operatorname{star}^{6}$ (half-filled circles) and linear ${ }^{16,17}$ (filled circles) polystyrenes in Figure 3. As the arm number increases, the $\log \left\langle S^{2}\right\rangle_{z}$ vs. $\log M_{w}$ relation parallely shifts down; note that the linear polymer corresponds 


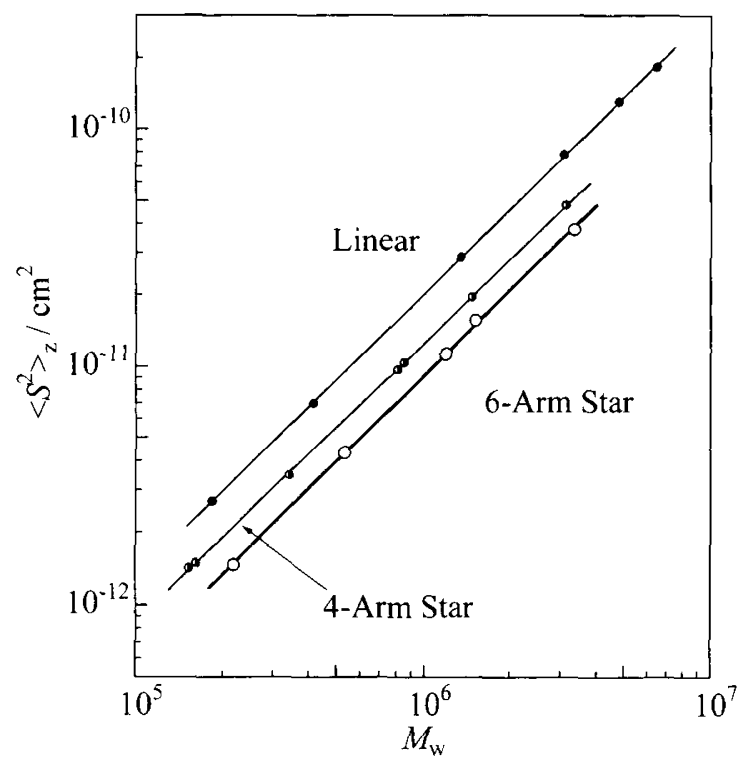

Figure 3. Molecular weight dependence of $\left\langle S^{2}\right\rangle_{z}$ for six-arm star polystyrene (O), four-arm star polystyrene ${ }^{6}(\mathbf{D})$, and linear polystyrene ${ }^{16,17}(\mathrm{)})$ in benzene at $25^{\circ} \mathrm{C}$.

to $f=2$. The straight line for the six-arm star polymer is expressed by

$$
\left.<S^{2}\right\rangle_{z}=6.6 \times 10^{-19} M_{w}^{1.19} \quad \text { (six-arm star) }
$$

in units of $\mathrm{cm}^{2}$. This is combined with the relation $\left\langle S^{2}\right\rangle_{z}=1.4_{7} \times 10^{-18} M_{w}^{1.19}\left(\mathrm{~cm}^{2}\right)$ for linear polystyrene in benzene (the uppermost straight line in the figure) to give 0.45 as the ratio $g_{S}$ of $\left\langle S^{2}\right\rangle_{z}$ for six-arm star polystyrene to that for the linear polymer with the same molecular weight, i.e., $g_{S}=\left\langle S^{2}\right\rangle_{z}\left(\right.$ six-arm star) $/\left\langle S^{2}\right\rangle_{z}$ (linear). This ratio agrees substantially with Roovers and Bywater's value $\mathrm{e}^{2,18} 0.47$ in toluene, another good solvent for polystyrene, and also with results (about 0.45) from renormalization group theory ${ }^{2,19}$ and Monte Carlo simulations. ${ }^{9,20}$ These ratios in the good solvent limit do not differ much from the experimental values $0.46-0.49$ for the same polymer in cyclohexane at the $\Theta$ point. ${ }^{4,18}$ They are even closer to Zimm and Stockmayer's theoretical value ${ }^{21} 0.444$ for Gaussian six-arm stars (see eq 8), as already observed for star polymers with $f \geq 6$ by previous workers. ${ }^{2,3}$

Figure 4 compares the molecular weight dependence of $A_{2}$ for six-arm star polystyrene in benzene at $25^{\circ} \mathrm{C}$ with that for four-arm star $^{6}$ and linear polystyrenes ${ }^{16,17}$ in the same solvent. The curve appears systematically lower for larger $f$. The negative slope for the six-arm star polymer is slightly steeper than those $(-0.25)$ for the other polymers in the $M_{w}$ range examined, but above $M_{w}$ $\sim 10^{6}$, the three lines are almost parallel, yielding 0.72 for the ratio $A_{2}$ (six-arm star)/ $A_{2}$ (linear). Figure 5 shows that $A_{3}$ is also systematically smaller for larger $f$. The three curves for the respective polymers ${ }^{6,22}$ are essentially linear and parallel with slope 0.6 for $M_{w}$ above $5 \times$ $10^{5}$, and the ratio $A_{3}$ (six-arm star)/A $A_{3}$ (linear) in the high molecular weight region is estimated to be 0.68 .

In Figure 6, the reduced third virial coefficients $\Gamma(=$ $A_{3} / A_{2}^{2} M_{w}$ ) for the three types of polystyrene in benzene

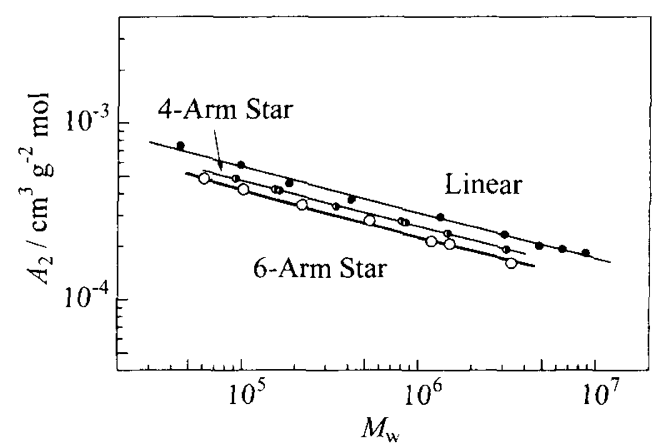

Figure 4. Molecular weight dependence of $A_{2}$ for six-arm star polystyrene $(\bigcirc)$, four-arm star polystyrene ${ }^{6}(\bigcirc)$, and linear polystyrene ${ }^{16,17}(O)$ in benzene at $25^{\circ} \mathrm{C}$.

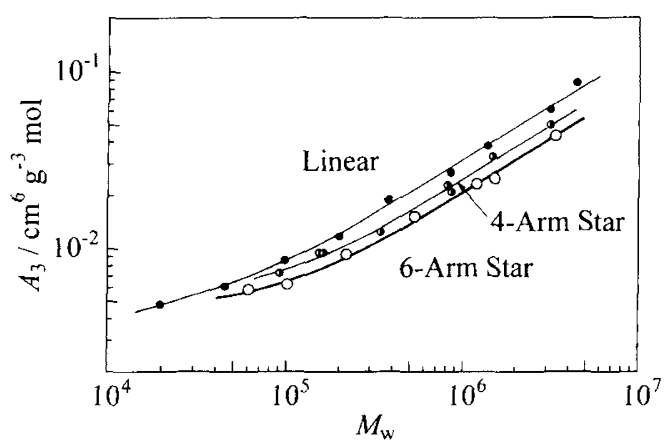

Figure 5. Molecular weight dependence of $A_{3}$ for six-arm star polystyrene $(\bigcirc)$, four-arm star polystyrene ${ }^{6}(O)$, and linear polystyrene ${ }^{15,22}(O)$ in benzene at $25^{\circ} \mathrm{C}$.

are plotted against $\log M_{w}$. As $f$ increases, $\Gamma$ at a fixed $M_{w}$ becomes larger, suggesting that its increase toward the value 0.625 for rigid spheres ${ }^{23}$ is due to that in segment density. It should be noted, however, that in the region of $M_{w}$ above $3 \times 10^{5}, \Gamma$ for each polymer is also an increasing function of molecular weight ; $\Gamma$ is roughly proportional to $M_{w}^{0.1}$ since $A_{2} \propto M_{w}^{-0.25}$ and $A_{3} \propto M_{w}^{0.6}$ for high $M_{w}$. Norisuye $e t a l{ }^{24}$ theoretically explained the dependence of $\Gamma$ on $\alpha_{S}{ }^{3}$ and hence on $M_{w}$ for linear polystyrene by incorporating effects of chain stiffness and three-segment interactions on $A_{3}$ into the classical mean-field theory of Stockmayer and Cassassa. ${ }^{25}$ According to their analysis, ${ }^{24}$ these effects are significant only for low $M_{w}$ where $\Gamma$ stays almost constant or even increases with decreasing $M_{w}$. Thus, the larger $\Gamma$ for larger $f$ observed at high $M_{w}$ may be considered a reflection of the difference in molecular architecture, and ought to be explained by a more sophisticated theory.

The molecular weight dependence of $[\eta]$ for six-arm star polystyrene in benzene is shown in Figure 7, which includes typical data for four-arm $\operatorname{star}^{6}$ and linear polystyrenes ${ }^{17,26}$ in benzene. As is the case with $\left\langle S^{2}\right\rangle_{z}, A_{2}$, and $A_{3},[\eta]$ lowers with increasing arm number. The straight line for the six-arm star polymer is represented by

$$
[\eta]=4.7 \times 10^{-5} M_{w}^{0.75} \quad(6 \text {-arm star })
$$

in units of $10^{2} \mathrm{~cm}^{3} \mathrm{~g}^{-1}$. Combining this with the relation $[\eta]=8.2 \times 10^{-5} M_{w}^{0.75}\left(10^{2} \mathrm{~cm}^{3} \mathrm{~g}^{-1}\right)$ for the linear poly- 


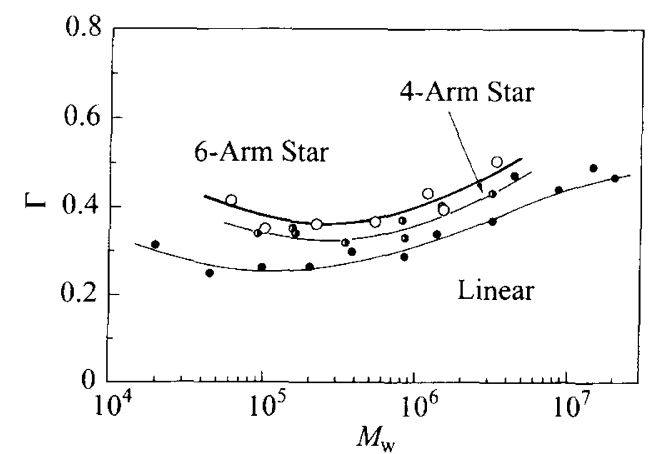

Figure 6. Plots of $\Gamma$ vs. $M_{w}$ for six-arm star polystyrene $(O)$, four-arm star polystyrene ${ }^{6}(\mathbf{C})$, and linear polystyrene ${ }^{15,22}(\boldsymbol{O})$ in benzene at $25^{\circ} \mathrm{C}$.

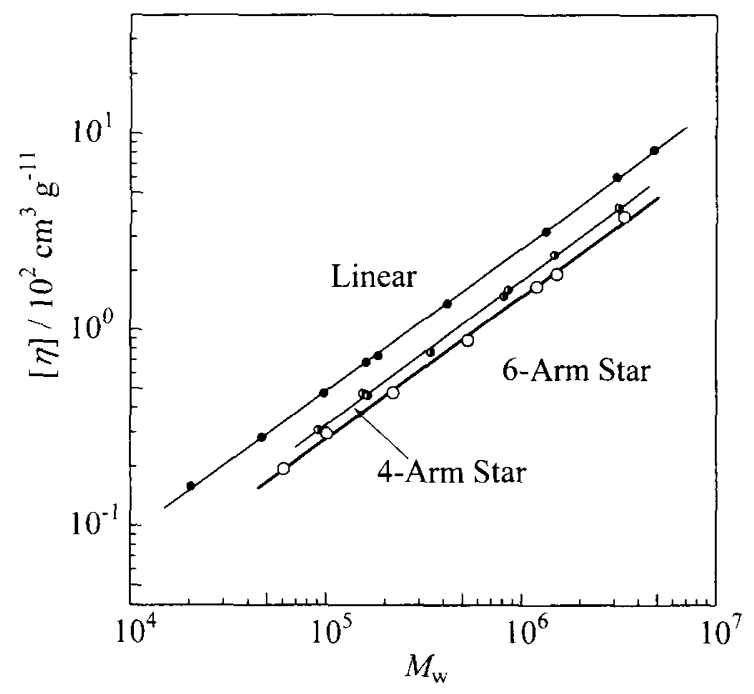

Figure 7. Molecular weight dependence of $[\eta]$ for six-arm star polystyrene $(\bigcirc)$, four-arm star polystyrene ${ }^{6}(()$ ), and linear polystyrene ${ }^{17,26}(\mathbf{O})$ in benzene at $25^{\circ} \mathrm{C}$.

mer, we obtain a value of 0.57 for the ratio $g_{\eta}[\equiv[\eta]$ (six-arm star) $/[\eta]$ (linear)], which is slightly smaller than our previous value 0.63 in cyclohexane at the $\Theta$ point. ${ }^{4}$ Such differences in $g_{\eta}$ between good and $\Theta$ solvents are known for various star polymers. ${ }^{2,3}$

Values of the Flory viscosity factor $\Phi\left[=[\eta] M_{w} /(6\right.$ $\left.\left.\left\langle S^{2}\right\rangle_{z}\right)^{3 / 2}\right]$ for six-arm star polystyrene were calculated to be $(3.6 \pm 0.3) \times 10^{23} \mathrm{~mol}^{-1}$ from the data in Table I. Note that since $[\eta] \propto M_{w}{ }^{0.75}$ and $\left\langle S^{2}\right\rangle_{z} \propto M_{w}{ }^{1.19}$ for the system, $\Phi$ is almost independent of $M_{w}$ in the limited range from $2.19 \times 10^{5}$ to $3.35 \times 10^{6}$. Our $\Phi$ of $3.6 \times 10^{23}$ $\mathrm{mol}^{-1}$ is somewhat smaller than the values $3.7 \times 10^{23}$ $4.2 \times 10^{23} \mathrm{~mol}^{-1}$ in toluene reported by Douglas et al., ${ }^{2}$ but it is quite close to the Monte Carlo result $\left(3.6 \times 10^{23}\right.$ $-3.7 \times 10^{23}$ ) of Shida et al. ${ }^{9}$ These $\Phi$ values are much larger than what is known for linear ${ }^{16,17,26}$ and four-arm star $^{6}$ polystyrenes (about $2.1 \times 10^{23} \mathrm{~mol}^{-1}$ and $2.8 \times 10^{23}$ $\mathrm{mol}^{-1}$, respectively) with comparable molecular weights in benzene at $25^{\circ} \mathrm{C}$, reflecting the difference in molecular architecture.

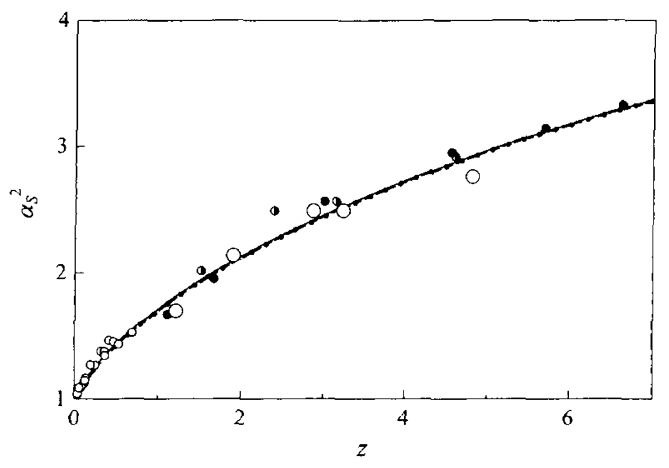

Figure 8. Plots of $\alpha_{S}^{2}$ vs. $z$ for six-arm star polystyrene in benzene (O) and cyclohexane ${ }^{4}(0)$, four-arm star polystyrene in benzene ${ }^{6}$ (C), and linear polystyrene in benzene ${ }^{16,17}($ ). The solid, dashed, and dotted lines represent eq 5 for $f=6,4$, and 2 , respectively.

\section{DISCUSSION}

\section{Expansion Factors}

As discussed previously, ${ }^{4}$ both $\alpha_{S}{ }^{2}$ and $\alpha_{\eta}^{3}$ (the expansion factor for $[\eta]$ ) for six-arm star polystyrene may be treated approximately in the conventional twoparameter theory ${ }^{27,28}$ corresponding to the coil limit of the quasi-two-parameter scheme ${ }^{29}$ for the helical wormlike chain, provided that $M_{w}$ is higher than $1.2 \times 10^{6}$. In this limit, these expansion factors should be universal functions of $z$ defined by

$$
z=\left(\frac{3}{2 \pi b^{2}}\right)^{3 / 2} \beta n^{1 / 2}
$$

where $\beta$ is the binary-cluster integral for the interaction between a pair of beads, $n$ is the total number of beads in the chain, and $b$ is the bead spacing. In the analyses below, we take a monomeric unit as one bead and make use of all the $\left\langle S^{2}>\right.$ and [ $\left.\eta\right]$ data given in Table I, though the effects of chain stiffness on them may not always be negligible for $M_{w}<10^{6}$.

With Miyaki's $\beta$ value of $3.0 \times 10^{-23} \mathrm{~cm}^{3}$ for linear polystyrene in benzene $\mathrm{e}^{16,17,28}$ and our previous $\left\langle S^{2}\right\rangle_{z}$ data $^{4}$ for six-arm star polystyrene samples $6 \mathrm{~S} 85,6 \mathrm{~S} 190$, $6 \mathrm{~S} 260$, and $6 \mathrm{~S} 570$ in cyclohexane at the $\Theta$ temperature $\left(34.5^{\circ} \mathrm{C}\right)$, we calculated $\alpha_{S}{ }^{2}$ and $z$ in benzene ; as in our previous work on four-arm star polystyrene, ${ }^{5,6}$ we ignored the possible difference between the unperturbed dimensions in benzene and cyclohexane. The resulting relation is shown by larger unfilled circles in Figure 8, along with the previously determined relation for the six-arm star polymer in cyclohexane near the $\Theta$ point ${ }^{4}$ (smaller unfilled circles) and those for four-arm star ${ }^{6}$ (half-filled circles) and linear ${ }^{16,17}$ (filled circles) polystyrenes in benzene. For clarity, cyclohexane data for the four-arm star and linear polymers, which almost overlap those for the six-arm star polymer, ${ }^{4}$ are not shown here. The experimental relations for the three polymers in benzene are hardly distinguishable within experimental error, as was found previously for cyclohexane solutions.

The previously proposed interpolation expression ${ }^{5}$ of $\alpha_{S}{ }^{2}$ for regular star polymers is

$$
\alpha_{S}^{2}=\left(1+5.56 K_{f} z+10.62 z^{20 / 9}\right)^{0.18}
$$




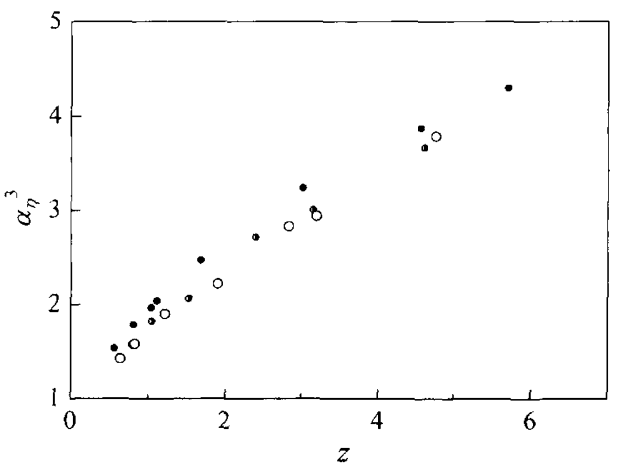

Figure 9. Plots of $\alpha_{y}^{3}$ vs. $z$ for six-arm star polystyrene $(\bigcirc)$, fourarm star polystyrene ${ }^{6}\left(()\right.$, and linear polystyrene ${ }^{17,26}(O)$ in benzene.

which was derived by combining the first-order perturbation theory ${ }^{7}$ and the asymptotic relation ${ }^{9} \alpha_{S}^{2}=1.53 z^{2 / 5}$ in the limit of infinite $z$. Here, $K_{f}$ is the first-order perturbation coefficient given by

$$
\begin{aligned}
K_{f}=\frac{6}{f^{1 / 2}(3 f-2)}\{ & \frac{67}{315}+\frac{268 \sqrt{2}-67}{315}(f-1) \\
& \left.+\frac{202 \sqrt{2}-276}{315}(f-1)(f-2)\right\}
\end{aligned}
$$

Equation 5 with eq 6 for $f=2$ gives $\alpha_{S}^{2}$ that agrees with the familiar Domb-Barrett equation ${ }^{30}$ within $1.3 \%$ for $z>0$. The solid, dashed, and dotted lines in Figure 8 represent eq 5 for $f=6,4$, and 2 , respectively. They come indistinguishably close to one another and fit the experimental points for the star and linear polymers in benzene as well as those in cyclohexane. Thus, we may conclude that eq 5 gives a fairly satisfactory description to the $z$-dependence of $\alpha_{S}^{2}$ for the three types of polymer of high molecular weight. Note that the stiffness effect on $\alpha_{S}^{2}$ is not very significant in the molecular weight range examined.

Figure 9 shows the $\alpha_{\eta}^{3}$ vs. $z$ relations for six-arm star, four-arm star, and linear polystyrenes in benzene, where, as in the case of $\alpha_{S}^{2}$, the [ $\left.\eta\right]$ data in cyclohexane at the $\Theta$ point have been used for the unperturbed intrinsic viscosities in benzene. The plotted points for the six-arm and four-arm star polymers appear slightly but systematically below those for the linear polymer. This difference in $\alpha_{\eta}^{3}$, at least for $z>2$, is a direct consequence of the smaller $g_{\eta}$ for the star polymers in benzene than in cyclohexane at $\Theta$. For $z<1$ the stiffness effect on $\alpha_{\eta}^{3}$ may not be negligible, since we previously observed no such difference in the $\alpha_{\eta}^{3} v s . z$ relation among the three polymers of high molecular weight in cyclohexane at different temperatures where $z$ was smaller than $0.7 .^{4}$ In any event, the $\alpha_{n}^{3}$ vs. $z$ relations for four-arm and sixarm star polystyrenes remain to be explained theoretically.

\section{Interpenetration Function}

Figure 10 shows the $\Psi$ data plotted against $\alpha_{S}{ }^{3}$ for six-arm star, four-arm star, and linear polystyrenes in benzene (the larger symbols) and those for high molecular weight samples of the three polymers in cyclohexane

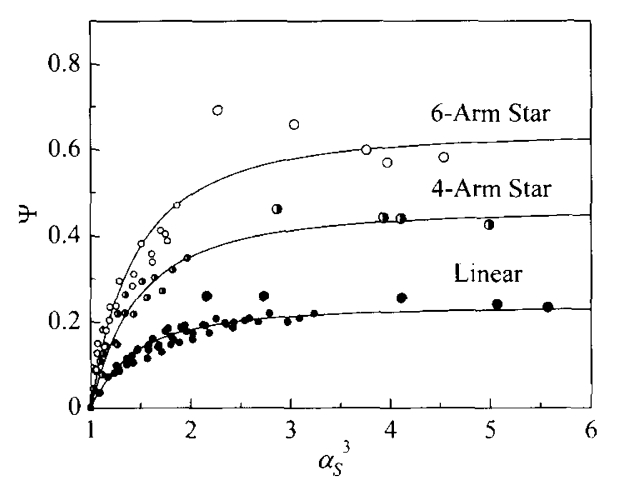

Figure 10. Plots of $\Psi$ vs. $\alpha_{S}^{3}$ for six-arm star polystyrene in benzene $(\bigcirc)$ and cyclohexane $e^{4}(\bigcirc)$, four-arm star polystyrene in benzene $^{6}(\mathbf{O})$ and cyclohexane $e^{5}(\mathbf{O})$, and linear polystyrene in benzene $^{16,17}\left(\right.$ ) and cyclohexane $\mathrm{e}^{16,17}(\bullet)$. The curves represent the values calculated from eq 7 with eq 5 for $f=6,4$, and 2 , respectively. The value of $\alpha_{S}{ }^{3}(=0.22$ ) for $6 S 36$ in benzene was obtained with the aid of the unperturbed mean-square radius of gyration estimated indirectly by linear extrapolation of the previously established $\log \left\langle S^{2}\right\rangle_{z}$ us. $\log M_{w}$ relation for six-arm star polystyrene in cyclohexane at the $\Theta$ point.

near the $\Theta$ temperature (the smaller symbols). Here, $\Psi$ is defined by $\Psi \equiv A_{2} M_{w}{ }^{2} /\left(4 \pi^{3 / 2} N_{A}<S^{2}>_{z}^{3 / 2}\right)$, with $N_{A}$ being the Avogadro constant, and the cyclohexane data are the reproductions from previous work. ${ }^{5,17}$ As the arm number increases, $\Psi$ shifts up, reflecting an increase in segment density. A fearure common to the three polymers is that, while $\Psi$ in cyclohexane near $\Theta$ is a sharply increasing function of $\alpha_{S}{ }^{3}$, that in the good solvent is a gradually decreasing function of it. For the six-arm star polymer, $\Psi$ appears to decrease to a value of about 0.6 , which agrees rather well with recent Monte Carlo data $(0.62-0.64) .{ }^{31,32}$ A similar agreement between experiment and Monte Carlo simulation was previously found for four-arm star polystyrene in benzene. ${ }^{6}$

The curves in Figure 10 represent the theoretical values calculated for the three types of polystyrene from our interpolation equation ${ }^{5}$

$$
\Psi=\frac{z}{\left(\alpha_{S}^{2} g_{S 0}\right)^{3 / 2}}\left[1+5 C_{f} z+\frac{0.0411}{\left(\Psi^{*} g_{S 0} 3 / 2\right)^{5}} z^{2}\right]^{-0.2}
$$

and eq 5 for $\alpha_{S}{ }^{2}$. Here, $C_{f}$ is the first-order perturbation coefficient ${ }^{8}$ for $A_{2}(5.39,3.87$, and 2.865 for $f=6,4$, and 2 , respectively), $\Psi^{*}$ is the value of $\Psi$ in the limit of $z \rightarrow$ $\infty(0.64,0.44$, and 0.235 for $f=6,4$, and 2 , respectively), ${ }^{10,31,32}$ and $g_{S 0}$ is the value of $g_{S}$ in the unperturbed state given by ${ }^{21}$

$$
g_{S 0}=(3 f-2) / f^{2}
$$

The general agreement between the calculated and experimental $\Psi$ is fairly good, allowing us to conclude that eq 7 is capable of consistently explaining the behavior of $\Psi$ for the three types of polymer.

It cannot be overlooked, however, that the present benzene data tend to deviate upward from the theoretical curve with decreasing $\alpha_{S}{ }^{3}$. This deviation is similar to or even more pronounced than those for four-arm $\operatorname{star}^{6}$ and linear flexible polymers ${ }^{16,28,29,33-37}$ in good sol- 
vents, and may be ascribed primarily to the effect of chain stiffness on $A_{2} \cdot{ }^{29,36}$ To obtain a better agreement, incorporation of this effect into eq 6 seems necessary.

\section{CONCLUSIONS}

The present data of $\left\langle S^{2}\right\rangle_{z}, A_{2}, A_{3}$, and [ $\eta$ ] for six-arm star polystyrene samples with $M_{w}$ of $6.1 \times 10^{4}$ to $3.4 \times 10^{6}$ in benzene at $25^{\circ} \mathrm{C}$ have been compared with the known results for four-arm star $^{6}$ and linear ${ }^{16,17,22,26}$ polystyrenes in the same solvent. The difference in molecular architecture among the three types of polystyrene manifests itself in the molecular weight dependence of each property and more distinctly in the interpenetration function $\Psi$ and Flory's viscosity factor. The relations between $\alpha_{S}{ }^{2}$ and $z$ are essentially the same for the three polymers of high molecular weight and explained by the previously constructed interpolation formula ${ }^{5}$ over a broad $z$ range. On the other hand, the curves of $\alpha_{\eta}{ }^{3} v$ s. $z$ for the four-arm and six-arm star polymers come slightly but systematically below that for the linear chain, differing from the previous observation ${ }^{4}$ for cyclohexane solutions at small $z$ where the three curves were essentially superimposed. Our $\Psi$ for the six-arm star polymer in benzene tends to decrease with increasing $M_{w}$ or $\alpha_{S}^{3}$ and approaches a value close to Monte Carlo results $(0.62-$ $0.64)$. Though the previous interpolation formula fairly well reproduces the $\Psi$ data in the good solvent, it fails to explain the gradual decrease in experimental $\Psi$ with increasing $\alpha_{S}{ }^{3}$ probably owing to the neglect of the stiffness effect on $A_{2}$.

\section{REFERENCES}

1. S. Bywater, Adv. Polym. Sci., 30, 89 (1979).

2. J. F. Douglas, J. Roovers, and K. F. Freed, Macromolecules, 23, 4168 (1990).

3. G. S. Grest, L. J. Fetters, J. S. Huang, and D. Richter, Adv. Chem. Phys., 94, 67 (1996).

4. M. Okumoto, Y. Tasaka, Y. Nakamura, and T. Norisuye, Macromolecules, 32, 7430 (1999).

5. M. Okumoto, K. Terao, Y. Nakamura, T. Norisuye, and A. Teramoto, Macromolecules, 30, 7493 (1997).

6. M. Okumoto, Y. Nakamura, T. Norisuye, and A. Teramoto, Macromolecules, 31, 1615 (1998).

7. G. C. Berry and T. A. Orofino, J. Chem. Phys., 40, 1614 (1964).
8. E. F. Casassa, J. Chem. Phys., 37, 2176 (1962).

9. K. Shida, M. Kimura, Y. Kawazoe, K. Ohno, and Y. Nakamura, Macromolecules, 31, 2343 (1998).

10. A. J. Barrett, Macromolecules, 18,196 (1985).

11. See, for example, T. Norisuye and Y. Nakamura, Macromolecules, 27, 2054 (1994).

12. B. J. Cherayil, J. F. Douglas, and K. F. Freed, J. Chem. Phys., 87, 3089 (1987).

13. G. C. Berry, J. Chem. Phys., 44, 4550 (1966).

14. C. E. H. Bawn, R. F. J. Freeman, and A. Kamaliddin, Trans. Faraday Soc., 46, 862 (1950).

15. T. Sato, T. Norisuye, and H. Fujita, J. Polym. Sci., Part B : Polym. Phys., 25, 1 (1987).

16. Y. Miyaki, Y. Einaga, and H. Fujita, Macromolecules, 11, 1180 (1978).

17. Y. Miyaki, Ph. D. Thesis, Osaka University, 1981.

18. J. E. L. Roovers and S. Bywater, Macromolecules, 7, 443 (1974).

19. J. F. Douglas and K. F. Freed, Macromolecules, 17, 2344 (1984).

20. A. J. Barret and D. L. Tremain, Macromolecules, 20, 1687 (1987).

21. B. H. Zimm and W. H. Stockmayer, J. Chem. Phys., 17, 1301 (1949).

22. Y. Nakamura, T. Norisuye, and A. Teramoto, J. Polym. Sci., Part B : Polym. Phys., 29, 153 (1991).

23. W. Burchard, Makromol. Chem., Macromol. Symp., 39, 179 (1990).

24. T. Norisuye, Y. Nakamura, and K. Akasaka, Macromolecules, 26, 3791 (1993).

25. W. H. Stockmayer and E. F. Casassa, J. Chem. Phys., 20, 1560 (1952).

26. Y. Einaga, Y. Miyaki, and H. Fujita, J. Polym. Sci., Polym. Phys. Ed., 17, 2103 (1979).

27. H. Yamakawa, "Modern Theory of Polymer Solutions", Harper \& Row, New York, N.Y., 1971.

28. H. Fujita, "Polymer Solutions", Elsevier, Amsterdam, 1990.

29. H. Yamakawa, "Helical Wormlike Chains in Polymer Solutions", Springer, Berlin, 1997.

30. C. Domb and A. J. Barret, Polymer, 17, 361 (1976).

31. K. Ohno, K. Shida, M. Kimura, and Y. Kawazoe, Macromolecules, 29, 2269 (1996).

32. A. M. Rubio and J. J. Freire, Macromolecules, 29, 6946 (1996).

33. Y. Miyaki, Y. Einaga, T. Hirosye, and H. Fujita, Macromolecules, 10, 1356 (1977).

34. K. Huber and W. H. Stockmayer, Macromolecules, 20, 1400 (1987).

35. Y. Nakamura, K. Akasaka, K. Katayama, T. Norisuye, and A. Teramoto, Macromolecules, 25, 1134 (1992).

36. H. Yamakawa, Macromolecules, 25, 1912 (1992).

37. H. Yamakawa, F. Abe, and Y. Einaga, Macromolecules, 26, 1898 (1993). 